Bull. Austral. Math. Soc.

VOL. 58 (1998) [307-332]

\title{
STABILITY OF DISCRETE ORTHOGONAL PROJECTIONS FOR CONTINUOUS SPLINES
}

\author{
R.D. GRIGORIEFF AND I.H. SLOAN
}

In this paper $L_{p}$ stability and convergence properties of discrete orthogonal projections on the finite element space $S_{h}$ of continuous polynomial splines of order $r$ are proved. The discrete inner products are defined by composite quadrature rules with positive weights on a sequence of nonuniform grids. It is assumed that the basic quadrature rule $Q$ has at least $r$ quadrature points in order to resolve $S_{h}$, but no accuracy is required. The main results are derived under minimal further assumptions, for example the rule $Q$ is allowed to be non-symmetric, and no quasi-uniformity of the mesh is required. The corresponding stability of the orthogonal $L_{2}$-projections has been studied by de Boor [1] and by Crouzeix and Thomée [2]. Stability of the first derivative of the projection is also proved, under an assumption (unless $p=1$ ) of local quasi-uniformity of the mesh.

\section{INTRODUCTION}

This paper establishes stability and convergence properties of discrete orthogonal projections onto the standard finite element spaces $S_{h}$ of continuous polynomial splines of degree at most $r-1$ on an interval, with $r \geqslant 2$.

To be precise, let

$$
\pi_{h}^{\prime}:=\left\{0=x_{0}<x_{1}<\cdots<x_{n}=L\right\}
$$

be an arbitrary partition of the interval $[0, L]$. Then

$$
S_{h}:=\left\{\psi \in C[0, L]: \psi \mid\left[x_{k}, x_{k+1}\right] \in \mathbb{P}_{r-1}, \quad k=0, \cdots, n-1\right\}
$$

where $\psi \mid[x, y]$ denotes the restriction of $\psi$ to $[x, y]$ and $\mathbb{P}_{d}$ the space of polynomials of degree at most $d$. Let

$$
Q g:=\sum_{j=1}^{J} w_{j} g\left(\xi_{j}\right) \sim \int_{0}^{1} g(x) d x
$$

Received 3rd March, 1998

The support of the Australian Research Council is gratefully acknowledged, as is the hospitality of the Mittag-Leffler Institute where the present version of this work was completed.

Copyright Clearance Centre, Inc. Serial-fee code: 0004-9729/98 \$A2.00+0.00. 
be a basic quadrature rule with quadrature points or nodes

$$
0 \leqslant \xi_{1}<\xi_{2}<\cdots<\xi_{J} \leqslant 1
$$

and weights $w_{j}>0$; the positivity of the weights plays a crucial role in the theory. We denote by $h$ the mesh-size vector

$$
h:=\left(h_{0}, h_{1}, \cdots, h_{n-1}\right),
$$

where $h_{k}:=x_{k+1}-x_{k}$ for $k=0, \cdots, n-1$, and introduce the composite quadrature rule

$$
Q_{h} g:=\sum_{k=0}^{n-1} h_{k} \sum_{j=1}^{J} w_{j} g\left(x_{k, j}\right) \sim \int_{0}^{L} g(x) d x,
$$

with

$$
x_{k, j}:=x_{k}+h_{k} \xi_{j}, \quad k=0, \cdots, n-1, \quad j=1, \cdots, J .
$$

We then introduce the positive semidefinite, Hermitian, sesquilinear form

$$
(f, g)_{h}:=Q_{h}(f \bar{g}), \quad f, g \in C[0, L] .
$$

This sesquilinear form is shown in Proposition 2.1 to be an inner product on $S_{h}$ if and only if $J \geqslant r$. In this situation we may define a projection $R_{h}: C[0, L] \rightarrow S_{h}$ by

$$
\left(R_{h} f, \psi\right)_{h}=(f, \psi)_{h} \quad \forall \psi \in S_{h} .
$$

It is this discrete orthogonal projection $R_{h}$ that we study in this paper.

One of our main resuits (see Theorems 5.1 and 4.4) is that for each $p \in[1, \infty]$, and for $h_{\max }:=\max _{k} h_{k}$, we have

$$
\left\|R_{h} f-f\right\|_{L_{p}(0, L)} \rightarrow 0 \text { as } h_{\max } \rightarrow 0 \quad \forall f \in C[0, L],
$$

and also, for $\ell=1, \cdots, r$,

$$
\left\|R_{h} f-f\right\|_{L_{p}(0, L)} \leqslant C h_{\max }^{\ell}\left\|f^{(\ell)}\right\|_{L_{p}(0, L)}, f \in W_{p}^{\ell}(0, L),
$$

under only the most basic assumptions on the quadrature rule: we do not even insist that the rule be symmetric. On the other hand, for all $J \geqslant r$ it is sufficient that $Q$ be symmetric; and if $J=r$ and $Q$ is not symmetric it is sufficient that $\xi_{1}=0$ and $\xi_{J}=1$. There are no other constraints on the choice of quadrature points, and (apart from positivity) no constraints on the weights. Observe in (1.6) and (1.7) that $R_{h}$ is defined with the aid of point evaluation of $f$, while the convergence holds in the $L_{p}$ norm. These convergence results require no restriction of any kind on the partition $\pi_{h}^{\prime}$, provided $h_{\max } \rightarrow 0$. 
The quadrature rule (1.3), and the sesquilinear form (1.4), can also be defined in a natural way on the space $G_{h}$ of 'grid functions', that is, of the complex-valued functions on the grid

$$
\pi_{h}:=\left\{x_{k, j}: k=0, \cdots, n-1, j=1, \cdots, J\right\}
$$

(where we use the convention that $x_{k, J}$ is considered different from $x_{k+1,1}$ even if their values coincide, which of course happens if $\xi_{J}=1$ and $\xi_{1}=0$ ). The convergence properties (1.6) and (1.7) rest on a corresponding stability property

$$
\left\|R_{h} f\right\|_{L_{p}(0, L)} \leqslant C\|f\|_{h, p}, \quad f \in C[0, L] \text { or } G_{h},
$$

where

$$
\begin{aligned}
\|f\|_{h, p} & :=Q_{h}\left(|f|^{p}\right)^{1 / p}, \quad f \in C[0, L] \text { or } G_{h}, \quad p \in[1, \infty), \\
\|f\|_{h, \infty} & :=\max \left\{\left|f\left(x_{k, j}\right)\right|: \quad k=0, \cdots, n-1, j=1, \cdots, J\right\} .
\end{aligned}
$$

Here and throughout the paper $C$ denotes a generic constant independent of $h$ and other significant quantities.

In Theorem 6.1 we state a stability result for the derivative $\left(R_{h} f\right)^{\prime}$, in the same setting, but this time with a quasi-uniformity assumption on the grid unless $p=1$.

In the final section, Section 7, we give results, again for rather general rules $Q$ and arbitrary meshes, for the stability of the discrete orthogonal projection $R_{h}^{0}$ on the space $S_{h}^{0} \subset S_{h}$, which is obtained from $S_{h}$ by imposing zero boundary conditions. The main results for $R_{h}^{0}$ are contained in Theorem 7.2. It turns out that $R_{h}^{0}$ shares the main features of $R_{h}$, and also has some additional new properties.

The continuous counterpart of (1.9) for the orthogonal $L_{2}$-projection $P_{h}$ on $S_{h}$ without any restriction on the partition $\pi_{h}^{\prime}$ has first been proved by Dupont (see de Boor [1]), extending earlier work of Douglas, Dupont and Wahlbin [5] for quasiuniform grids. The proof in [1] relies essentially on the total positivity of the Gram matrix for the B-spline basis of $S_{h}$. It carries over directly to our discrete case if the quadrature rule is symmetric, but its extension to the non-symmetric case, and to the study of the first derivative of the projection, is less obvious. In the present paper we follow more closely the reasoning of Crouzeix and Thomée [2], who in turn based their arguments on the earlier work of Descloux [4]. Those papers are mainly concerned with higher dimensional problems, but the paper [2] also gives an explicit treatment of the continuous 1-dimensional problem that serves as a starting point for the present investigation. In that paper the stability of $P_{h}$ in the 1-dimensional case is related to the diagonal dominance of a tridiagonal matrix, in an argument that is more elementary and gives easier control of the constants determining stability than the total positivity argument. In our discrete case the corresponding way of reasoning enables us to consider also nonsymmetric rules $Q$. Thus, for example, the above-mentioned general case $J=r$ and $\xi_{1}=0, \xi_{J}=1$ is included, 
which is of special interest because then $R_{h}$ is an interpolatory projection or collocation projection for collocating in the points of $\pi_{h}$. The diagonal dominance argument also allows, as in [2], extension to a stability argument for the first derivative of $R_{h} f$.

It might be useful to remark that the extension of the 1-dimensional arguments of [2] to the discrete case is not entirely straightforward, since the proof of diagonal dominance of the tridiagonal matrix in the cited paper relies on being able to obtain explicitly, via integration by parts, a certain polynomial; and thence to integrate exactly certain integrals involving this polynomial. In our case the corresponding orthogonality is with respect to a discrete version of the inner product, so explicit knowledge of this kind is not available, not least because integration by parts is no longer valid. We may remark that for some other methods extension from the continuous to the discrete case is easier. In particular, as pointed out in [6], the extension is straightforward, under very general conditions, for the continuous-case arguments used in [5]. However the style of argument used in that work seems inevitably to impose restrictions on the mesh.

It is well known that the stability property of $P_{h}$ is the key to proving $L_{p}$ convergence results for finite element methods with trial space $S_{h}$ on nonuniform partitions; see for example [11]. In the same way (1.9) is basic to the analysis of Galerkin methods with quadrature. Some differential equation applications of (1.9) to a discrete Petrov-Galerkin method are given in $[\mathbf{9}, \mathbf{1 0}]$. For the (relatively trivial) special case $r=2$ some of the results obtained in this paper have been obtained previously in [9], where they were used to analyse a discrete Petrov-Galerkin method for boundary and eigenvalue problems associated with $m$-th order ordinary differential equations. Applications of (1.9) to certain non-linear differential and integro-differential problems are discussed in [8].

In this paper we do not treat $R_{h}$ as a small perturbation of the corresponding continuous orthogonal projection operator $P_{h}$, nor do we assume that the rule $Q$ has any polynomial degree of precision, as for example in the analysis of approximate Galerkin methods in [7, Theorem XII 1.15]. The admission of general quadrature formulas in this paper may lead to new classes of fully discrete methods for 1-dimensional differential, integro-differential and integral equation problems that are, in our view, of independent interest, while at the same time permitting analysis of some existing methods, ranging from collocation methods to perturbations of Galerkin-type methods.

\section{THE MAP $R_{h}$}

We begin by characterising conditions under which (1.4) is an inner product on $S_{h}$. In this case $R_{h}$ is well-defined by (1.5).

PROPOSITION 2.1. The positive semidefinite Hermitian form $(.,)_{h}$ defined in (1.4) is an inner product on $S_{h}$ if and only if $J \geqslant r$. 
Proof: Assume initially $J \geqslant r$, and let $\psi \in S_{h}$. Because the definition of $\|f\|_{h, 2}$ uses the values of $f$ only at the quadrature points, clearly, $\|\psi\|_{h, 2}=0$ if and only if

$$
\psi\left(x_{k, j}\right)=0, \quad k=0, \cdots, n-1, j=1, \cdots, J .
$$

Now consider $\psi \mid \triangle_{k}$, the restriction of $\psi$ to $\triangle_{k}$, where

$$
\triangle_{k}:=\left[x_{k}, x_{k+1}\right], k=0, \cdots, n-1 .
$$

Since $\psi \mid \triangle_{k} \in \mathbb{P}_{r-1}$, it is clear for $J \geqslant r$ that (2.1) can hold only if $\psi=0$. Thus $\|\psi\|_{h, 2}=0$ implies $\psi=0$ if $J \geqslant r$. Assume now $J \leqslant r-1$. Then there exists a polynomial $q \in \mathbb{P}_{r-1}, q \neq 0$, satisfying $q\left(\xi_{j}\right)=0, j=1, \cdots, J$. We define

$$
\psi(x):=\alpha_{k} q\left(\frac{x-x_{k}}{h_{k}}\right), \quad x \in\left(x_{k}, x_{k+1}\right), k=0, \cdots, n-1,
$$

and choose the constants $\alpha_{k}$, not all zero, in such a way that $\psi$ can be continued to a non-zero function in $C[0, L]$ which by construction vanishes at $x_{k, j}, k=0, \cdots, n-1$, $j=1, \cdots, J$. For example, if $q(0) \neq 0$, one can choose $\alpha_{0}=1$ and then define $\psi$ on consecutive intervals, beginning with the interval $\left(x_{0}, x_{1}\right)$. Similarly, in the case $q(0)=0$ but $q(1) \neq 0$ we start with $k=n-1$. If $q(0)=q(1)=0$, then we can take $\alpha_{k}=1$ for all $k$.

In accordance with this result we assume $J \geqslant r$ in the rest of this paper, so that $R_{h}$ is always well-defined.

The next step (following a line of argument in [2]) is to split the spline space $S_{h}$ into a direct sum

$$
S_{h}=S_{h, 1}+S_{h, 2}
$$

in such a way that $S_{h, 2}$ has a purely local basis (that is, with support in a single $\Delta_{k}$ only), and $S_{h, 1}$ is orthogonal to $S_{h, 2}$ with respect to the discrete inner product $(.,)_{h}$. The spaces in (2.4) are thus given by

$$
\begin{aligned}
& S_{h, 2}:=\left\{\psi \in S_{h}: \psi\left(x_{k}\right)=0, k=0, \cdots, n\right\}, \\
& S_{h, 1}:=\left\{\psi \in S_{h}:\left(\psi, \phi_{h}\right)_{h}=0 \forall \phi_{h} \in S_{h, 2}\right\} .
\end{aligned}
$$

The dimension of $S_{h, 1}$ is $\operatorname{dim} S_{h, 1}=n+1$, which is independent of $r$. The dimension of $S_{h, 2}$ is $n(r-2)$. (In the case $r=2$ one has $S_{h, 2}=\{0\}$ ).

We now construct a basis of $S_{h, 1}$. Let $\psi^{(i)} \in \mathbb{P}_{r-1}, i=0,1$, be the polynomials defined by

$$
\begin{aligned}
& Q\left(\psi^{(i)} \phi\right)=0 \quad \forall \phi \in \mathbb{P}_{r-1}^{o}, \\
& \psi^{(0)}(0)=1, \psi^{(0)}(1)=0, \psi^{(1)}(0)=0, \psi^{(1)}(1)=1,
\end{aligned}
$$

where $\mathbb{P}_{d}^{0}$ denotes the subspace of polynomials $\phi \in \mathbb{P}_{d}$ such that $\phi(0)=\phi(1)=0$. The uniqueness, and hence existence, of $\psi^{(i)}$ for fixed $i$ follows from the fact that according to 
(2.7) the difference $\psi$ of two candidate polynomials lies in $\mathbb{P}_{r-1}^{\circ}$ and satisfies $Q(\psi \phi)=0$ for all $\phi \in \mathbb{P}_{r-1}^{\circ}$, from which follows $Q\left(\psi^{2}\right)=0$, and hence $\psi=0$ since the polynomial $\psi$ is at most of degree $r-1$ and vanishes at the $r$ points $\xi_{1}, \cdots, \xi_{r}$. Note that only quadrature points lying in $(0,1)$ influence the definition of $\psi^{(0)}$ and $\psi^{(1)}$, since $\phi(0)=\phi(1)=0$. Note too that if the contribution to the quadrature formula from the open interval $(0,1)$ is symmetric then

$$
\psi^{(1)}(x)=\psi^{(0)}(1-x), x \in[0,1] .
$$

The polynomials $\psi^{(i)}$ are real, because the quadrature weights $w_{j}$ are real. Now let

$$
\begin{aligned}
& \psi_{0}(x):=\psi^{(0)}\left(\frac{x}{h_{0}}\right), x \in \triangle_{0}, \\
& \psi_{k}(x):=\left\{\begin{array}{cc}
\psi^{(1)}\left(\frac{x-x_{k-1}}{h_{k-1}}\right), & x \in \triangle_{k-1} \\
\psi^{(0)}\left(\frac{x-x_{k}}{h_{k}}\right), & x \in \triangle_{k}
\end{array}\right\}, k=1, \cdots, n-1, \\
& \psi_{n}(x):=\psi^{(1)}\left(\frac{x-x_{n-1}}{h_{n-1}}\right), x \in \triangle_{n-1},
\end{aligned}
$$

and zero elsewhere. Evidently, $\psi_{k} \in S_{h}, k=0, \cdots, n$, and $\psi_{k}\left(x_{\ell}\right)=\delta_{k \ell}, 0 \leqslant k, \ell \leqslant n$.

In the sequel we use the notation

$$
(f, g)_{h}^{(k)}:=h_{k} \sum_{j=1}^{J} w_{j} f\left(x_{k, j}\right) \bar{g}\left(x_{k, j}\right), k=0, \cdots, n-1,
$$

and the analogous notation $\|f\|_{h, p}^{(k)}$. Clearly we have

$$
(f, \dot{g})_{h}=\sum_{k=0}^{n-1}(f, g)_{h}^{(k)} .
$$

LEMMA 2.2. $\left\{\psi_{0}, \cdots, \psi_{n}\right\}$ is a basis for $S_{h, 1}$.

Proof: Since the $\psi_{k}$ are clearly linearly independent and $\operatorname{dim} S_{h, 1}=n+1$, the assertion is proved if we know $\psi_{k} \in S_{h, 1}$. We show this explicitly for the case $k \in[1, n-1]$ only. Choose any $\phi \in S_{h, 2}$. Then

$$
\left(\psi_{k}, \phi\right)_{h}=\left(\psi_{k}, \phi\right)_{h}^{(k-1)}+\left(\psi_{k}, \phi\right)_{h}^{(k)}
$$

where

$$
\begin{aligned}
\left(\psi_{k}, \phi\right)_{h}^{(k)} & =h_{k} \sum_{j=1}^{J} w_{j}\left(\psi_{k} \bar{\phi}\right)\left(x_{k}+h_{k} \xi_{j}\right) \\
& =h_{k} \sum_{j=1}^{J} w_{j} \psi^{(0)}\left(\xi_{j}\right) \bar{\phi}\left(x_{k}+h_{k} \xi_{j}\right)=0
\end{aligned}
$$


The last step follows from (2.7) because $\bar{\phi}\left(x_{k}+h_{k} \xi\right)$ as a function of $\xi$ is in $\mathbb{P}_{r-1}^{\infty}$. In the same way we can show that $\left(\psi_{k}, \phi\right)_{h}^{(k-1)}=0$. Thus $\left(\psi_{k}, \phi\right)_{h}=0$, and $\psi_{k} \in S_{h, 1}$. The results for $k=0$ and $n$ follow similarly.

The decomposition of $\psi \in S_{h}$ that corresponds to (2.4) is easily achieved, once the functions $\psi_{k}$ have been constructed.

Lemma 2.3. Assume $J \geqslant r$. An arbitrary $\psi \in S_{h}$ may be represented uniquely as

$$
\psi=\psi_{h, 1}+\psi_{h, 2}, \psi_{h, 1} \in S_{h, 1}, \psi_{h, 2} \in S_{h, 2}
$$

Moreover

$$
\psi_{h, 1}=\sum_{k=0}^{n} \psi\left(x_{k}\right) \psi_{k}
$$

Proof: The uniqueness is clear since (2.4) is an orthogonal decomposition. To prove existence, let $\psi \in S_{h}$ be given, and note that

$$
\sum_{k=0}^{n} \psi\left(x_{k}\right) \psi_{k} \in S_{h, 1}, \quad \psi-\sum_{k=0}^{n} \psi\left(x_{k}\right) \psi_{k} \in S_{h, 2}
$$

the latter holding because (on recalling $\psi_{k}\left(x_{\ell}\right)=\delta_{k \ell}$ ) the given expression vanishes at $x_{\ell}$ for $\ell=0, \cdots, n$. Thus $\psi_{h, 1}=\Sigma_{k} \psi\left(x_{k}\right) \psi_{k}$.

We now split the mapping $R_{h}$ according to the above splitting of $S_{h}$. To this end we define $R_{h, i}: G_{h} \rightarrow S_{h, i}$ for $i=1,2$ by

$$
\left(R_{h, i} f, \psi\right)_{h}=(f, \psi)_{h} \quad \forall \psi \in S_{h, i} .
$$

Note that $R_{h, 1}$ and $R_{h, 2}$ are well defined for $J \geqslant r$, since by Proposition $2.1(\cdot, \cdot)_{h}$ is an inner product on $S_{h, i} \subset S_{h}$.

LEMma 2.4. Assume $J \geqslant r$. Then $R_{h}=R_{h, 1}+R_{h, 2}$.

Proof: Any element $\psi \in S_{h}$ can be written as $\psi=\phi_{1}+\phi_{2}$ with $\phi_{\ell} \in S_{h, \ell} \ell=1,2$. Taking (2.6) into account it is seen that

$$
\begin{aligned}
\left(\left(R_{h, 1}+R_{h, 2}\right) f, \psi\right)_{h} & =\left(R_{h, 1} f+R_{h, 2} f, \phi_{1}+\phi_{2}\right)_{h} \\
& =\left(R_{h, 1} f, \phi_{1}\right)_{h}+\left(R_{h, 2} f, \phi_{2}\right)_{h},
\end{aligned}
$$

where the cross terms vanish. Thus

$$
\left(\left(R_{h, 1}+R_{h, 2}\right) f, \psi\right)_{h}=\left(f, \phi_{1}\right)_{h}+\left(f, \phi_{2}\right)_{h}=(f, \psi)_{h}=\left(R_{h} f, \psi\right)_{h},
$$

which with Proposition 2.1 proves the assertion.

If we represent $R_{h, 1} f$ in the form

$$
R_{h, 1} f=\sum_{\ell=0}^{n} c_{\ell} \psi_{\ell}
$$


then (2.11) for $i=1$ is equivalent to the linear system

$$
\sum_{\ell=0}^{n} A_{k \ell} c_{\ell}=b_{k}, \quad k=0, \cdots, n
$$

where we have introduced the scaled Gram matrix $A_{h}$ of the basis $\left\{\psi_{k}\right\}$, with elements

$$
A_{k \ell}:=\frac{\left(\psi_{k}, \psi_{\ell}\right)_{h}}{h_{k-1}+h_{k}}, k, \ell=0, \cdots, n
$$

and corresponding right-hand sides

$$
b_{k}:=\frac{\left(f, \psi_{k}\right)_{h}}{h_{k-1}+h_{k}}, k=0, \cdots, n
$$

with $h_{-1}:=h_{n}:=0$. Note that $R_{h, 2}=0$ if $r=2$, since in that case $S_{h, 2}=\{0\}$.

\section{Properties of $A_{h}$}

Our proof of the stability property (1.9) hinges on showing that under appropriate circumstances the tridiagonal matrix $A_{h}$ defined by (2.14) is row diagonally dominant; and moreover that the maximum difference of a diagonal element and the corresponding row sum of absolute values of the off-diagonal elements is bounded away from zero. It is convenient to establish these matrix properties immediately. The desired diagonal dominance result is Proposition 3.2. The result is re-expressed as an inverse stability property of $A_{h}$ in Proposition 3.6.

To simplify the statement of the following lemma, we define

$$
A_{0,-1}:=A_{n, n+1}:=0
$$

and introduce

$$
\alpha_{k}:=\frac{h_{k-1}}{h_{k}+h_{k-1}}, k=0, \cdots, n .
$$

Note that $0 \leqslant \alpha_{k} \leqslant 1$ for $k=0, \cdots, n$ and $\alpha_{0}=0, \alpha_{n}=1$.

LEMмA 3.1. The matrix $A_{h}$ is tridiagonal and has positive diagonal elements. Moreover, for $k=0, \cdots, n$ we have

$$
\begin{aligned}
\sigma_{h, k}:= & A_{k k}-\left|A_{k, k-1}\right|-\left|A_{k, k+1}\right| \\
= & \alpha_{k} Q\left(\psi^{(1) 2}\right)+\left(1-\alpha_{k}\right) Q\left(\psi^{(0) 2}\right)-\left|Q\left(\psi^{(1)} \psi^{(0)}\right)\right| \\
= & \frac{1}{2} Q\left(\left|\psi^{(1)}-\psi^{(0)} \operatorname{sgn}\left(Q\left(\psi^{(1)} \psi^{(0)}\right)\right)\right|^{2}\right) \\
& +\left(\alpha_{k}-\frac{1}{2}\right)\left(Q\left(\psi^{(1) 2}\right)-Q\left(\psi^{(0) 2}\right)\right)
\end{aligned}
$$

where $\operatorname{sgn} t:=1$ or -1 for $t \geqslant 0$ or $t<0$, respectively. 
ProOF: By definition of $\psi_{k}$, one calculates $\left(\psi_{k}, \psi_{\ell}\right)_{h}=0$ for $|k-\ell| \geqslant 2$, and

$$
\begin{aligned}
\left(\psi_{k}, \psi_{k}\right)_{h} & =h_{k-1} Q\left(\psi^{(1) 2}\right)+h_{k} Q\left(\psi^{(0) 2}\right) \\
\left(\psi_{k}, \psi_{k-1}\right)_{h} & =h_{k-1} Q\left(\psi^{(1)} \psi^{(0)}\right) \\
\left(\psi_{k}, \psi_{k+1}\right)_{h} & =h_{k} Q\left(\psi^{(1)} \psi^{(0)}\right) .
\end{aligned}
$$

It follows that

$$
\begin{aligned}
A_{k k} & =\alpha_{k} Q\left(\psi^{(1) 2}\right)+\left(1-\alpha_{k}\right) Q\left(\psi^{(0) 2}\right) \\
A_{k, k-1} & =\alpha_{k} Q\left(\psi^{(1)} \psi^{(0)}\right) \\
A_{k, k+1} & =\left(1-\alpha_{k}\right) Q\left(\psi^{(1)} \psi^{(0)}\right)
\end{aligned}
$$

from which (3.1) is obtained immediately.

In the rest of the paper we consider a sequence $H$ of gridsize vectors $h$ such that $h_{\max } \rightarrow 0(h \in H)$ and corresponding grids $\pi_{h}^{\prime}, h \in H$.

LEMMA 3.2. For each $h \in H$ let

$$
\sigma_{h}:=\min \left\{\sigma_{h, k}: k=0, \cdots, n\right\}
$$

Then

$$
\sigma_{h}=\sigma:=\min \left(Q\left(\psi^{(1) 2}\right), Q\left(\psi^{(0) 2}\right)\right)-\left|Q\left(\psi^{(1)} \psi^{(0)}\right)\right|, \quad h \in H .
$$

ProOF: From (3.1) we obtain immediately $\sigma_{h, k} \geqslant \sigma, k=0, \cdots, n$. Equality holds because $\alpha_{0}=0, \alpha_{n}=1$.

The following proposition states sufficient conditions for the existence of a suitable positive number bounding $\sigma_{h, k}$ below, that is, for $\sigma>0$. In other cases $\sigma>0$ can be tested by computation of $\sigma$ from the representation (3.2).

Proposition 3.3. Assume $J \geqslant r$. Sufficient conditions for the constant $\sigma$ defined in (3.2) to satisfy $\sigma>0$ are:

(a) $Q\left(\psi^{(1) 2}\right)=Q\left(\psi^{(0) 2}\right)$

(b) $Q$ is symmetric

(c) $Q$ integrates exactly all $p \in \mathbb{P}_{2 \tau-4}$ with respect to the weight function $w(x):=x(1-x)$, that is,

$$
\int_{0}^{1} p(x) w(x) d x=Q(p w), \quad p \in \mathbb{P}_{2 r-4}
$$

(d) $Q\left(\psi^{(1)} \psi^{(0)}\right)=0$

(e) $J=r$ and $\xi_{1}=0, \xi_{J}=1$

(f) $\min \left(Q\left(\psi^{(1)}\right), Q\left(\psi^{(0)}\right), Q\left((2 x-1) \psi^{(1)}\right), Q\left((1-2 x) \psi^{(0)}\right)\right)>0$. 
Proof: If $Q\left(\psi^{(1) 2}\right)=Q\left(\psi^{(0) 2}\right)$ then it follows from Lemma 3.2 and the last form of (3.1) that

$$
\sigma=\frac{1}{2} Q\left(\left|\psi^{(1)}-\psi^{(0)} \operatorname{sgn}\left(Q\left(\psi^{(1)} \psi^{(0)}\right)\right)\right|^{2}\right) \geqslant 0,
$$

with equality possible only if $\psi:=\psi^{(1)}-\psi^{(0)} \operatorname{sgn}\left(Q\left(\psi^{(1)} \psi^{(0)}\right)\right)$ vanishes at each quadrature point. Since $J \geqslant r$ and $\psi \in \mathbb{P}_{r-1}$ this implies $\psi=0$, contradicting $\psi(1)=1$.

If $Q$ is symmetric then we have observed already that $\psi^{(1)}(x)=\psi^{(0)}(1-x)$, from which $Q\left(\psi^{(1) 2}\right)=Q\left(\psi^{(0) 2}\right)$ follows. Thus this case is covered by case (a).

If $Q$ integrates exactly all polynomials of degree at most $2 r-4$ with respect to the weight function $w$ then the condition $Q\left(\psi^{(i)} \phi\right)=0$ for all $\phi \in \mathbb{P}_{r-1}^{\circ}$ in the definition (2.7) can be replaced by the corresponding integral (since $\phi(x)=x(1-x) \tau(x)$ with $\tau \in \mathbb{P}_{r-3}$ ), from which it follows that $\psi^{(1)}(x)=\psi^{(0)}(1-x)$, so that again the case is covered by (a).

If $Q\left(\psi^{(1)} \psi^{(0)}\right)=0$ the result follows immediately from Lemma 3.2, since $Q\left(\psi^{(1) 2}\right) \geqslant$ 0 , with equality excluded because $\psi^{(1)}$ vanishing at all quadrature points would imply $\psi^{(1)}=0$, contradicting $\psi^{(1)}(1)=1$. Thus case (d) is proved.

If $J=r$ and $\xi_{1}=0, \xi_{J}=1$ then there are exactly $J-2=r-2$ interior nodes $\xi_{2}, \cdots, \xi_{J-1}$ for the rule $Q$. Let $\left\{\phi_{j}: j=2, \cdots, J-1\right\} \subset \mathbb{P}_{r-3}$ be the set of fundamental Lagrange polynomials for the $J-2$ interior nodes, that is,

$$
\phi_{j} \in \mathbb{P}_{r-3}, \phi_{j}\left(\xi_{j^{\prime}}\right)=\delta_{j j^{\prime}}, 2 \leqslant j, j^{\prime} \leqslant J-1 .
$$

Then $x(1-x) \phi_{j}(x) \in \mathbb{P}_{r-1}^{o}$, and from (2.7) we have

$$
Q\left(x(1-x) \psi^{(i)} \phi_{j}\right)=0, \quad j=2, \cdots, J-1, \quad i=0,1,
$$

which implies, given (3.4) and $w_{j}>0$, that

$$
\psi^{(i)}\left(\xi_{j}\right)=0 \text { for } j=2, \cdots, J-1, \quad i=0,1,
$$

so that $\psi^{(i)}$ vanishes at each interior node. Since also $\psi^{(0)}(0)=\psi^{(1)}(1)=1$ and $\psi^{(0)}(1)=$ $\psi^{(1)}(0)=0$ it follows that $Q\left(\psi^{(1)} \psi^{(0)}\right)=0$, thus the result for case (e) follows from case (d).

Turning to case (f) we note that

$$
\begin{aligned}
Q\left(\psi^{(1) 2}\right)-\left|Q\left(\psi^{(1)} \psi^{(0)}\right)\right| & =Q\left(\psi^{(1) 2}-\psi^{(1)} \psi^{(0)} \operatorname{sgn}\left(Q\left(\psi^{(1)} \psi^{(0)}\right)\right)\right) \\
& =Q\left(\psi^{(1)}\left[\psi^{(1)}-\psi^{(0)} \operatorname{sgn}\left(Q\left(\psi^{(1)} \psi^{(0)}\right)\right)\right]\right)
\end{aligned}
$$

Now observe that

$$
\begin{aligned}
\psi^{(1)}+\psi^{(0)}-1 & \in \mathbb{P}_{r-1}^{o}, \\
\psi^{(1)}-\psi^{(0)}-(2 x-1) & \in \mathbb{P}_{r-1}^{o} .
\end{aligned}
$$


On writing $\psi^{(1)}+\psi^{(0)}=1+\phi$ and $\psi^{(1)}-\psi^{(0)}=(2 x-1)+\psi$, with $\phi, \psi \in \mathbb{P}_{r-1}^{\infty}$, it follows from $Q\left(\psi^{(1)} \phi\right)=Q\left(\psi^{(1)} \psi\right)=0$ that

$$
Q\left(\psi^{(1) 2}\right)-\left|Q\left(\psi^{(1)} \psi^{(0)}\right)\right|= \begin{cases}Q\left(\psi^{(1)}\right) & \text { if } Q\left(\psi^{(1)} \psi^{(0)}\right)<0 \\ Q\left(\psi^{(1)}(2 x-1)\right) & \text { if } Q\left(\psi^{(1)} \psi^{(0)}\right) \geqslant 0\end{cases}
$$

An analogous result holds with $\psi^{(1)}$ replaced by $\psi^{(0)}$ if $2 x-1$ is replaced by $1-2 x$. This proves case (f).

Remark 3.4. The condition (c) in Proposition 3.3 is satisfied, for instance, by the $(r-1)$-point Gauss-Jacobi rule belonging to the weight $x(1-x)$ (see [3, Section 2.7]) together with an adjoined quadrature point $\xi_{r}:=1$ (to bring the total number of points up to $r$ ), with any positive number allowed for $\omega_{r}$. It is also satisfied by any rule which is exact for polynomials of degree at most $2 r-2$, such as the $r$-point Radau rules (which are unsymmetric, see [3, Section 2.7.1]), or the $r$-point Gauss-Legendre rule.

REMARK 3.5. If condition (e) of Proposition 3.3 is satisfied it can be seen from the proof that $Q\left(\psi^{(0) 2}\right)=w_{1}, Q\left(\psi^{(1) 2}\right)=w_{J}$ and hence $\sigma=\min \left(w_{1}, w_{J}\right)$.

REMARK 3.6. If there are at least $r-2$ interior nodes in $Q$ then the functions $\psi^{(i)}, i=$ 0,1 , are already well-defined, and hence $A_{h}$ is well-defined. Thus under this assumption it makes sense to consider the possibility of diagonal dominance of $A_{h}$ even for $J=r-2$ and $J=r-1$.

The following results can be shown. If $J=r-2$ and all nodes are interior then $A_{h}=0$. If $J=r-1$ and there are exactly $r-2$ interior nodes then $\sigma=0$. If all $J=r-1$ nodes are interior, and if also $Q$ is symmetric then $\sigma_{h, k}=0, k=0, \cdots, n$, and $A_{h}$ is singular. For $Q$ not symmetric still $\sigma \leqslant 0$ holds in this case. (Proofs of these results can be obtained on the basis of (7.15) and (3.1).)

Definition 3.7: Given $p \in[1, \infty]$, the sequence of matrices $\left\{A_{h}\right\}_{H}$ is said to be inversely $p$-stable if

$$
\gamma_{p}:=\inf \left\{\left|A_{h} c\right|_{h, p}: c \in G_{h}^{\prime},|c|_{h, p}=1, h \in H\right\}>0
$$

where $G_{h}^{\prime}$ denotes the vector space of grid functions defined on $\pi_{h}^{\prime}$, and

$$
\begin{aligned}
|c|_{h, p} & :=\left(\sum_{k=0}^{n} \frac{h_{k-1}+h_{k}}{2}\left|c_{k}\right|^{p}\right)^{1 / p}, p \in[1, \infty) \\
|c|_{h, \infty} & :=\max _{k=0, \cdots, n}\left|c_{k}\right|=:|c|_{\infty} .
\end{aligned}
$$

The next lemma shows that it is sufficient to establish the inverse $p$-stability of $\left\{A_{h}\right\}_{H}$ for $p=1$ or $p=\infty$ in order to obtain it for all $p$.

LEMMA 3.8. For $p \in(1, \infty), \gamma_{1}=\gamma_{\infty} \leqslant \gamma_{p}$. 
ProOF: For the vector $\tilde{c} \in \mathbb{C}^{n+1}$ with components

$$
\tilde{c}_{k}:=\frac{h_{k-1}+h_{k}}{2} c_{k}, \quad k=0, \cdots, n,
$$

we have $|c|_{h, 1}=|\tilde{c}|_{1}$, and after a short calculation it is also verified that

$$
\left|A_{h} c\right|_{h, 1}=\left|A_{h}^{*}\right|_{1},
$$

where $A_{h}^{*}$ denotes the adjoint matrix of $A_{h},|\cdot|_{p}$ is the usual $\ell_{p}$ norm and $|A|_{p}$ denotes the matrix norm induced by the vector norm $|\cdot|_{p}$. Because $A_{h}$ is square, it is clear that $A_{h}^{*}$ is injective if and only if $A_{h}$ is injective, and that in the case they are injective

$$
\left|\left(A_{h}^{*}\right)^{-1}\right|_{1}=\left|A_{h}^{-1}\right|_{\infty}
$$

Consequently, if we introduce

$$
\gamma_{h, p}:=\inf \left\{\left|A_{h}\right|_{h, p}: c \in G_{h}^{\prime},|c|_{h, p}=1\right\}, \quad h \in H,
$$

we have either $\gamma_{h, 1}=\gamma_{h, \infty}=0$ or

$$
\gamma_{h, \infty}=\left|A_{h}^{-1}\right|_{\infty}^{-1}=\left|\left(A_{h}^{*}\right)^{-1}\right|_{1}^{-1}=\gamma_{h, 1}, \quad h \in H .
$$

On taking the infimum over $h \in H$ this shows $\gamma_{1}=\gamma_{\infty}$. And $\gamma_{1} \leqslant \gamma_{p}$ then follows by an application of the Riesz-Thorin interpolation theorem.

Now we use Lemma 3.2 to obtain a result for the inverse $p$-stability of $\left\{A_{h}\right\}_{H}$.

Propositrion 3.9. Assume $\sigma>0$ in (3.2). Then $\left\{A_{h}\right\}_{H}$ is inversely $\infty$-stable with $\gamma_{\infty} \geqslant \sigma$. If any of the conditions (a)-(c) in Proposition 3.3 is satisfied then $\gamma_{\infty}=\sigma$.

Proof: It follows from Lemma 3.2 that $\sigma_{h, k} \geqslant \sigma$ for $k=0, \cdots, n$. For given $c \in \mathbb{C}^{n+1}$, let $j$ be such that $\left|c_{j}\right|=|c|_{\infty}$. Then with $c_{-1}:=c_{n+1}:=0$ we have

$$
\begin{aligned}
\mid A_{h} c_{\infty} & \geqslant\left|A_{j j} c_{j}+A_{j, j-1} c_{j-1}+A_{j, j+1} c_{j+1}\right| \\
& \geqslant\left(A_{j j}-\left|A_{j, j-1}\right|-\left|A_{j, j+1}\right|\right)\left|c_{j}\right|=\sigma_{h, j} \mid c_{\infty}
\end{aligned}
$$

and so $\gamma_{\infty} \geqslant \sigma$. Any of the conditions (a)-(c) in Proposition 3.3 implies $Q\left(\psi^{(1) 2}\right)=$ $Q\left(\psi^{(0) 2}\right)$, and from (3.1) and Lemma 3.2 follows $\sigma_{h, k}=\sigma$ for $k=0, \cdots, n$. On choosing $c$ to be the vector with components

$$
c_{k}:=\left(-\operatorname{sgn}\left(Q\left(\psi^{(1)} \psi^{(0)}\right)\right)\right)^{k}, \quad k=0, \cdots, n,
$$

it is easily verified that $\left|A_{h} c\right|_{\infty}=\sigma|c|_{\infty}$; note the second inequality in (3.7), which in this case is an equality. Consequently $\gamma_{\infty} \leqslant \sigma$, and the proof is complete. 


\section{STABILITY of $\left\{R_{h}\right\}_{H}$ AND INVERSE STABILITY of $\left\{A_{h}\right\}_{H}$.}

In this section we establish the stability of $\left\{R_{h}\right\}_{H}$. The main result is Theorem 4.4. Let $p \in[1, \infty]$, and as before let $H$ denote any sequence of mesh-size vectors $h$ such that $h_{\max } \rightarrow 0$. The sequence $\left\{R_{h}\right\}_{H}$ is called $p$-stable if there exists a constant $C$ such that

$$
\left\|R_{h} f\right\|_{L_{p}(0, L)} \leqslant C\|f\|_{h, p}, \quad f \in G_{h}, \quad h \in H,
$$

and $p$-stability for $\left\{R_{h, i}\right\}_{H}, i=1,2$, is defined in the same way. The next lemma shows that $\left\{R_{h, 2}\right\}_{H}$ is always $p$-stable. The proof is straightforward because $R_{h, 2}$ has essentially a local definition. Recall that $R_{h, 2}=0$ if $r=2$.

LEMMA 4.1. Let $r \geqslant 3$ and let

$$
C_{1}:=\sup _{0 \neq q \in \mathbb{P}_{r-1}^{o}} \max \left\{\frac{1}{Q\left(|q|^{2}\right)} \int_{0}^{1}|q(\xi)| d \xi \max _{j=1, \cdots, J}\left|q\left(\xi_{j}\right)\right|, \frac{Q(|q|)}{Q\left(|q|^{2}\right)} \max _{\xi \in[0,1]}|q(\xi)|\right\} .
$$

Then, for all $p \in[1, \infty]$

$$
\left\|R_{h, 2} f\right\|_{L_{p}(0, L)} \leqslant C_{1}\|f\|_{h, p}, f \in G_{h}, h \in H .
$$

Proof: We first prove the result for $p=1$. Let $f \in G_{h}$ be given. Choose any $k \in[0, n-1]$, and define

$$
\psi(x):=R_{h, 2} f(x), \quad x \in \triangle_{k},
$$

and $\psi(x):=0$ elsewhere. Then by (2.5) $\psi \in S_{h, 2}$, and by the definition (2.11) of $R_{h, 2}$ we have

$$
(\psi, \psi)_{h}^{(k)}=(\psi, \psi)_{h}=\left(R_{h, 2} f, \psi\right)_{h}=(f, \psi)_{h}=(f, \psi)_{h}^{(k)},
$$

or

$$
\left(\|\psi\|_{h, 2}^{(k)}\right)^{2}=(f, \psi)_{h}^{(k)} \leqslant\|f\|_{h, 1}^{(k)}\|\psi\|_{h, \infty}^{(k)}
$$

and consequently, if $\psi \neq 0$,

$$
\left\|R_{h, 2} f\right\|_{L_{1}\left(\Delta_{k}\right)}=\|\psi\|_{L_{1}\left(\Delta_{k}\right)} \leqslant \frac{\|\psi\|_{L_{1}\left(\Delta_{k}\right)}\|\psi\|_{h, \infty}^{(k)}}{\left(\|\psi\|_{h, 2}^{(k)}\right)^{2}}\|f\|_{h, 1}^{(k)} \leqslant C_{1}\|f\|_{h, 1}^{(k)},
$$

where we have taken into account that $\psi$ restricted to the subinterval $\Delta_{k}=\left[x_{k}, x_{k+1}\right]$ is a polynomial of degree at most $r-1$ satisfying $\psi\left(x_{k}\right)=\psi\left(x_{k+1}\right)=0$. After summing with respect to $k$, inequality (4.2) is proved for $p=1$.

Similarly,

$$
\left(\|\psi\|_{h, 2}^{(k)}\right)^{2} \leqslant\|f\|_{h, \infty}^{(k)}\|\psi\|_{h, 1}^{(k)}
$$

and reasoning as in (4.3),

$$
\left\|R_{h, 2} f\right\|_{L_{\infty}\left(\Delta_{k}\right)}=\|\psi\|_{L_{\infty}\left(\Delta_{k}\right)} \leqslant C_{1}\|f\|_{h, \infty}^{(k)}
$$


follows, from which (4.2) for $p=\infty$ is derived. For the remaining values $p \in(1, \infty)$ the assertion is obtained by an application of the Riesz-Thorin interpolation theorem.

By virtue of Lemma 4.1 the $p$-stability of $\left\{R_{h}\right\}_{H}$ is equivalent to the $p$-stability of $\left\{R_{h, 1}\right\}_{H}$. As a first step towards proving this $p$-stability, we relate it to the inverse $p$-stability of the sequence $\left\{A_{h}\right\}_{H}$ from (2.12).

Lemma 4.2. Let $p \in[1, \infty]$. If $\left\{A_{h}\right\}_{H}$ is inversely $p$-stable then $\left\{R_{h}\right\}_{H}$ is $p$ stable.

Proof: According to Lemma 4.1 we have only to show the $p$-stability of $\left\{R_{h, 1}\right\}_{H}$. Recall the definition of the basis $\left\{\psi_{k}\right\}$ of $S_{h, 1}$. If $\chi_{k}$ denotes the characteristic function for the interval $\left(x_{k}-h_{k-1}, x_{k}+h_{k}\right)$ then

$$
\left|\left(f, \psi_{k}\right)_{h}\right| \leqslant\left\|\chi_{k} f\right\|_{h, p}\left\|\psi_{k}\right\|_{h, p^{\prime}},
$$

where $1 / p+1 / p^{\prime}=1$. Consider the case $p \in(1, \infty)$. Then

$$
\begin{aligned}
\left\|\psi_{k}\right\|_{h, p^{\prime}}^{p^{\prime}} & =h_{k-1} \sum_{j=1}^{J} w_{j}\left|\psi^{(1)}\left(\xi_{j}\right)\right|^{p^{\prime}}+h_{k} \sum_{j=1}^{J} w_{j}\left|\psi^{(0)}\left(\xi_{j}\right)\right|^{p^{\prime}} \\
& \leqslant C_{2}^{p^{\prime}}\left(h_{k-1}+h_{k}\right),
\end{aligned}
$$

where

$$
C_{2}:=\max _{i=0,1}\left(\sum_{j=1}^{J} w_{j}\left|\psi^{(i)}\left(\xi_{j}\right)\right|^{p^{\prime}}\right)^{1 / p^{\prime}} .
$$

Thus we obtain from (2.15) and (3.6)

$$
\begin{aligned}
|b|_{h, p}^{p} & =\sum_{k=0}^{n} \frac{h_{k-1}+h_{k}}{2}\left|\frac{\left(f, \psi_{k}\right)_{h}}{h_{k-1}+h_{k}}\right|^{p} \leqslant C_{2}^{p} \sum_{k=0}^{n} \frac{1}{2}\left(h_{k-1}+h_{k}\right)^{1+p / p^{\prime}-p}\left\|\chi_{k} f\right\|_{h, p}^{p} \\
& =C_{2}^{p} \sum_{k=0}^{n} \frac{1}{2}\left\|\chi_{k} f\right\|_{h, p}^{p} \leqslant C_{2}^{p} \sum_{k=0}^{n}\left(\|f\|_{h, p}^{(k)}\right)^{p}=C_{2}^{p}\|f\|_{h, p}^{p},
\end{aligned}
$$

where we used $1+p / p^{\prime}-p=p\left(1 / p+1 / p^{\prime}-1\right)=0$. Now let $c \in \mathbb{C}^{n+1}$ be the solution of (2.13). With the aid of the assumed inverse $p$-stability of $\left\{A_{h}\right\}_{H}$ we obtain

$$
\gamma_{p}|c|_{h, p} \leqslant\left|A_{h} c\right|_{h, p}=|b|_{h, p} \leqslant C_{2}\|f\|_{h, p} .
$$

An inspection of the proof shows that this estimate also holds in the cases $p=1$ and $p=\infty$. On recalling (2.12), the assertion now follows with the aid of the easily verified inequality

$$
\left\|R_{h, 1} f\right\|_{L_{p}(0, L)}=\left\|\sum_{k=0}^{n} c_{k} \psi_{k}\right\|_{L_{p}(0, L)} \leqslant C_{3}|c|_{h, p}
$$

where

$$
C_{3}:=2^{1 / p}\left(\int_{0}^{1}\left(\left|\psi^{(0)}(\xi)\right|^{p^{\prime}}+\left|\psi^{(1)}(\xi)\right|^{p^{\prime}}\right)^{p / p^{\prime}} d \xi\right)^{1 / p}
$$


the last step following by application of the Hölder inequality to the integrand on the right of the identity

$$
\left\|\sum_{k=0}^{n} c_{k} \psi_{k}\right\|_{L_{p}(0, L)}^{p}=\sum_{j=0}^{n-1} h_{j} \int_{0}^{1}\left|c_{j} \psi^{(0)}(\xi)+c_{j+1} \psi^{(1)}(\xi)\right|^{p} d \xi
$$

CoROLLARY 4.3. If $\left\{A_{h}\right\}_{H}$ is inversely $\infty$-stable then $\left\{R_{h}\right\}_{H}$ is $p$-stable for all $p \in[1, \infty]$ and the stability constant can be chosen independently of $p$.

Proof: Combining Lemmas 3.5 and 4.2, the sequence $\left\{R_{h}\right\}_{H}$ is seen to be $p$-stable for all $p \in[1, \infty]$. By tracing the dependence of the stability constant for $\left\{R_{h, 1}\right\}_{H}$ in the proof of Lemma 4.2 we see that it can be chosen to be independent of $p$, since $\gamma_{p} \geqslant \gamma_{\infty}$ and $C_{3}$ can be bounded independently of $p$. The stability constant $C_{1}$ for $\left\{R_{h, 2}\right\}_{H}$ is also independent of $p$.

Now we are able to state our main result on the $p$-stability of $\left\{R_{h}\right\}_{H}$.

THEOREM 4.4. Assume $J \geqslant r$ and $\sigma>0$ in (3.2). Then $\left\{R_{h}\right\}_{H}$ is $p$-stable for $p \in[1, \infty]$, with the stability constant able to be chosen independently of $p$.

Proof: Combine Proposition 3.6 with Corollary 4.3.

Before leaving this section, we note the corresponding $p$-stability result that comes from replacing the quadrature rule $Q$ in $(1.2)$ by the exact integral $I$. In this case $(\cdot, \cdot)_{h}$ is replaced by the $L_{2}$ inner product

$$
(f, g):=\int_{0}^{L} f(x) \bar{g}(x) d x
$$

the projection $R_{h}$ defined by (1.5) is replaced by the $L_{2}$-orthogonal projection $P_{h}$ on $S_{h}$, and the norm $\|\cdot\|_{h, p}$ on the space of grid functions defined by (1.10) becomes the $L_{p}$ norm $\|\cdot\|_{L_{p}(0,1)}$. The following theorem is essentially [2, Theorem 1], except that the earlier work used in place of $S_{h}$ the subspace $S_{h}^{0}$ of functions with zero boundary conditions. It is also an easy consequence of $[1$, Theorem 2$]$.

THEOREM 4.5. Let $P_{h}$ be the $L_{2}$-orthogonal projection on $S_{h}$, and let $p \in[1, \infty]$. Then

$$
\left\|P_{h} g\right\|_{L_{p}(0, L)} \leqslant C\|g\|_{L_{p}(0, L)}, \quad g \in L_{p}(0, L), h \in H,
$$

where $C$ does not depend on $p$.

A proof could mirror the proof of (4.1), if we use the correspondences above, and note that the integral has all the properties of a symmetric quadrature rule with $J \geqslant r$. It is also possible to give a proof based on Theorem 4.4. We omit the details. 


\section{Convergence Properties of $\left\{R_{h}\right\}_{H}$.}

Theorem 5.1. Let $p \in[1, \infty]$ and let $\left\{R_{h}\right\}_{H}$ be $p$-stable. Then

$$
\begin{aligned}
\left\|R_{h} f\right\|_{L_{p}(0, L)} & \leqslant C\|f\|_{h, \infty}, f \in G_{h}, h \in H \\
\left\|R_{h} g-g\right\|_{L_{p}(0, L)} & \rightarrow 0(h \in H), g \in C[0, L] \\
\left\|R_{h} g-g\right\|_{L_{p}(0, L)} & \leqslant C h_{\max }^{\ell}\left\|g^{(\ell)}\right\|_{L_{p}(0, L)}, g \in W_{p}^{\ell}(0, L)
\end{aligned}
$$

for $\ell=1, \cdots, r$.

PROOF: The estimate (5.1) is an immediate consequence of the $p$-stability of $\left\{R_{h}\right\}_{H}$ defined in (4.1), since

$$
\|f\|_{h, p} \leqslant\left(L \sum_{j=1}^{J} w_{j}\right)^{1 / p}\|f\|_{h, \infty} .
$$

Since $R_{h} g_{h}=g_{h}$ for $g_{h} \in S_{h}$, the convergence property (5.2) follows from

$$
\begin{aligned}
\left\|R_{h} g-g\right\|_{L_{p}(0, L)} & \leqslant\left\|R_{h}\left(g-g_{h}\right)\right\|_{L_{p}(0, L)}+\left\|g-g_{h}\right\|_{L_{p}(0, L)} \\
& \leqslant C\left\|g-g_{h}\right\|_{h, p}+\left\|g-g_{h}\right\|_{L_{p}(0, L)}
\end{aligned}
$$

if we choose $g_{h}$ to be piecewise linear interpolant of $g$ interpolating at the breakpoints $x_{0}, \cdots, x_{n}$, which satisfies

$$
\left\|g-g_{h}\right\|_{C[0, L]} \rightarrow 0(h \in H) .
$$

For the proof of (5.3), for given $g \in W_{p}^{\ell}(0, L)$ use the same argument, but with $g_{h} \in S_{h}$ the interpolating function from Lemma $A 1$ or $A 2$ for $\ell=1$ or $\ell>1$ respectively.

\section{AN ESTIMATE FOR THE DERIVATIVE OF $R_{h} f$}

In $[2$, Theorem 2] the bound

$$
\left\|\left(P_{h} g\right)^{\prime}\right\|_{L_{p}(0, L)} \leqslant C\left\|g^{\prime}\right\|_{L_{p}(0, L)}, g \in \stackrel{\circ}{W}_{p}^{1}(0, L),
$$

for $p \in[1, \infty]$, has been proved for the orthogonal projection $P_{h}$ on $S_{h}^{0}$. It is shown in [2] that some restriction has to be imposed on the nonuniformity of the partition $\pi_{h}^{\prime}$ for (6.1) to hold, unless $p=1$. We shall assume that $\pi_{h}^{\prime}$ is locally quasiuniform, that is, for some constant $\gamma>1$ the condition

$$
\gamma^{-1} \leqslant \frac{h_{k-1}}{h_{k}} \leqslant \gamma, k=1, \cdots, n-1, h \in H
$$

holds, and that with a constant $\delta \geqslant 1$ to be specified later,

$$
\frac{h_{k-1}+h_{k}}{h_{\ell-1}+h_{\ell}} \leqslant C \delta^{|k-\ell|}, k, \ell=0, \cdots, n .
$$


In [2] a similar bound to that in (6.3) is assumed to hold for $i_{k} / h_{\ell}$ instead. It was pointed out by de Boor [1] that the weaker assumption (6.3) is sometimes advantageous.

In the space of grid functions $G_{h}$ on $\pi_{h}$ we introduce the mapping $D_{h}$,

$$
D_{h} f\left(x_{k, j}\right):=h_{k}^{-1} f\left(x_{k, j}\right), j=1, \cdots, J, k=0, \cdots, n-1 .
$$

We shall prove the following.

Theorem 6.1. Let $p \in[1, \infty]$. Assume that $\sigma>0$ in (3.2). Then

$$
\rho:=\frac{\left|Q\left(\psi^{(1)} \psi^{(0)}\right)\right|}{\min \left(Q\left(\psi^{(1) 2}\right), Q\left(\psi^{(0) 2}\right)\right)}<1 .
$$

If $p>1$ assume additionally that $\pi_{h}^{\prime}$ is locally quasiuniform and that (6.3) holds with

$$
\delta<\rho^{-p /(p-1)} .
$$

Then

$$
\left\|\left(R_{h} f\right)^{\prime}\right\|_{L_{p}(0, L)} \leqslant C\left\|D_{h} f\right\|_{h, p}, f \in G_{h}, h \in H .
$$

REMARK 6.2. The estimate (6.7) takes a more familiar form if we apply it to a grid function

$$
f:=g-g_{h},
$$

where $g \in W_{p}^{1}(0, L)$ and $g_{h}$ is the piecewise linear interpolant of $g$ in the breakpoints $x_{0}, \cdots, x_{n}$. With the aid of (A1) we then obtain

$$
\begin{aligned}
\left\|D_{h}\left(g-g_{h}\right)\right\|_{h, p}^{p} & =\sum_{k=0}^{n-1} h_{k} \sum_{j=1}^{J} w_{j} h_{k}^{-p}\left|\left(g-g_{h}\right)\left(x_{k, j}\right)\right|^{p} \\
& \leqslant C \sum_{k=0}^{n-1}\left\|g^{\prime}\right\|_{L_{p}\left(\Delta_{k}\right)}^{p}=C\left\|g^{\prime}\right\|_{L_{p}(0, L)}^{p} .
\end{aligned}
$$

(Here we assume $p \in[1, \infty)$, but the corresponding result is easily seen to hold also for $p=\infty$.) Since $R_{h} g_{h}=g_{h}$, we obtain from (6.7),

$$
\begin{aligned}
\left\|\left(R_{h} g\right)^{\prime}\right\|_{L_{p}(0, L)} & \leqslant\left\|\left(R_{h}\left(g-g_{h}\right)\right)^{\prime}\right\|_{L_{p}(0, L)}+\left\|g_{h}^{\prime}\right\|_{L_{p}(0, L)} \\
& \leqslant C\left\|g^{\prime}\right\|_{L_{p}(0, L)}
\end{aligned}
$$

for $g \in W_{p}^{1}(0, L), h \in H$, where the known estimate $\left\|g_{h}^{\prime}\right\|_{L_{p}(0, L)} \leqslant\left\|g^{\prime}\right\|_{L_{p}(0, L)}$ was used.

We prepare for the proof of Theorem 6.1 with some lemmas.

LEMmA 6.3. The following estimate holds for $p \in[1, \infty]$ : 


$$
\left\|\left(R_{h, 2} f\right)^{\prime}\right\|_{L_{p}(0, L)} \leqslant C\left\|D_{h} f\right\|_{h, p}, f \in G_{h}, h \in H .
$$

PRoOF: Since $R_{h, 2} f \mid \triangle_{k} \in \mathbb{P}_{r-1}^{\circ}$ and vanishes at $x_{k}$ and $x_{k+1}$, the inverse estimate

$$
\left\|\left(R_{h, 2} f\right)^{\prime}\right\|_{L_{p}\left(\Delta_{k}\right)} \leqslant C_{3} h_{k}^{-1}\left\|R_{h, 2} f\right\|_{L_{p}\left(\Delta_{k}\right)}
$$

holds for $k=0, \cdots, n-1$, with

$$
C_{3}:=\max _{0 \neq q \in \mathbf{P}_{r-1}^{0}} \frac{\left\|q^{\prime}\right\|_{L_{p}(0,1)}}{\|q\|_{L_{p}(0,1)}} .
$$

The estimate (6.9) now follows with the aid of the piecewise version of Lemma 4.1,

$$
\left\|R_{h, 2} f\right\|_{L_{p}\left(\Delta_{k}\right)} \leqslant C\|f\|_{h, p}^{(k)}, f \in G_{h}
$$

after summing with respect to $k$ the $p$-th powers (for $p \in[1, \infty)$ ) of the resulting inequality.

In the next lemma we need the diagonal part of the matrix $A_{h}$,

$$
B_{h}:=\operatorname{diag}\left(A_{k k}\right) \text {, }
$$

and also

$$
E_{h}:=B_{h}^{-1}\left(A_{h}-B_{h}\right)
$$

LEMMA 6.4. If (6.5) holds then

$$
\left.\left.|| E_{h}\right|^{\ell}\right|_{p} \leqslant(2 \ell+1)^{1 / p} \rho^{\ell}, \ell \in \mathbb{N}, p \in[1, \infty] .
$$

Here $\left|E_{h}\right|$ denotes the matrix obtained by taking the absolute values of the elements in $E_{h}$, and as before, $\left|F_{h}\right|_{p}$ denotes the least upper bound norm of a matrix $F_{h}$ with respect to the usual $\ell_{p}$ norm.

PROOF: Observe that $E_{h}$ is tridiagonal with zero diagonal. In the proof of Lemma 3.1 we have calculated the $A_{k \ell}$. From there we see, using also (6.5) and $0 \leqslant \alpha_{k} \leqslant 1$, that

$$
\left|E_{h}\right|_{\infty}=\max _{k} \frac{\left|Q\left(\psi^{(1)} \psi^{(0)}\right)\right|}{\alpha_{k} Q\left(\left|\psi^{(1)}\right|^{2}\right)+\left(1-\alpha_{k}\right) Q\left(\left|\psi^{(0)}\right|^{2}\right)} \leqslant \rho,
$$

and hence

$$
\left.\left.|| E_{h}\right|^{\ell}\right|_{\infty} \leqslant\left|E_{h}\right|_{\infty}^{\ell} \leqslant \rho^{\ell}
$$

The matrix $E_{h}^{\ell}$ is a banded matrix with at most $(2 \ell+1)$ diagonals, from which it follows that

$$
\left.\left.|| E_{h}\right|^{\ell}\right|_{1} \leqslant\left.\left.(2 \ell+1)|| E_{h}\right|^{\ell}\right|_{\infty} \leqslant(2 \ell+1) \rho^{\ell},
$$

The assertion then follows by interpolation.

For the formulation of the next lemma we introduce the $(n+1) \times(n+1)$ diagonal matrix

$$
T_{h}:=\left(\operatorname{diag}\left(h_{k-1}+h_{k}\right)\right)_{k=0}^{n} .
$$


LEMMA 6.5. Under the assumptions of Theorem 6.1

$$
\left|\left(T_{h}^{-1 / p^{\prime}} A_{h} T_{h}^{1 / p^{\prime}}\right)^{-1}\right|_{p} \leqslant C, h \in H, p \in[1, \infty] .
$$

Proof: We have the representation

$$
\begin{aligned}
T_{h}^{-1 / p^{\prime}} A_{h} T_{h}^{1 / p^{\prime}} & =T_{h}^{-1 / p^{\prime}}\left(B_{h}+A_{h}-B_{h}\right) T_{h}^{1 / p^{\prime}} \\
& =B_{h}\left(I_{h}+T_{h}^{-1 / p^{\prime}} E_{h} T_{h}^{1 / p^{\prime}}\right)
\end{aligned}
$$

where $I_{h}$ is the identity matrix. Since the diagonal elements of $A_{h}$ and hence also of $B_{h}$ satisfy

$$
\begin{aligned}
A_{k k} & =\alpha_{k} Q\left(\left|\psi^{(1)}\right|^{2}\right)+\left(1-\alpha_{k}\right) Q\left(\left|\psi^{(0)}\right|^{2}\right) \\
& \geqslant \min \left(Q\left(\left|\psi^{(1)}\right|^{2}\right), Q\left(\left|\psi^{(0)}\right|^{2}\right)\right)>0,
\end{aligned}
$$

it is easily seen that $\left|B_{h}^{-1}\right|_{p} \leqslant C$. So (6.12) is proved if we show that the Neumann series for $\left(I_{h}+T_{h}^{-1 / p^{\prime}} E_{h} T_{h}^{1 / p^{\prime}}\right)^{-1}$ has, uniformly with respect to $h \in H$, a convergent majorant in the $p$ norm. That this is in fact the case is seen from the following estimate, which takes (6.3) and Lemma 6.4 into account, together with the $(2 \ell+1)$-diagonal nature of $E_{h}^{\ell}$,

$$
\begin{aligned}
\left|T_{h}^{-1 / p^{\prime}} E_{h}^{\ell} T_{h}^{1 / p^{\prime}}\right|_{p} & \leqslant\left.\left.\max _{|i-k| \leqslant \ell}\left(\frac{h_{i-1}+h_{i}}{h_{k-1}+h_{k}}\right)^{1 / p^{\prime}}|| E_{h}\right|^{\ell}\right|_{p} \\
& \leqslant C(2 \ell+1)^{1 / p}\left(\delta^{1 / p^{\prime}} \rho\right)^{\ell},
\end{aligned}
$$

where $\delta^{1 / p^{\prime}} \rho<1$ due to condition (6.6). Thus the Neumann series converges as desired, and the result is proved.

PROOF OF THEOREM 6.1: In view of Lemma 6.3 it is sufficient to prove the bound for $\left(R_{h, 1} f\right)^{\prime}$ only. Now $R_{h, 1} f$ can be written in the form

$$
R_{h, 1} f=\sum_{\ell=0}^{n} c_{\ell} \psi_{\ell},
$$

where the vector $c=\left(c_{0}, \cdots, c_{n}\right)$ is determined by the linear system (2.13). Then with $c_{-1}:=c_{n+1}:=0$, since $\left.\psi_{\ell}\right|_{\Delta_{j}}=0$ unless $\ell=j$ or $\ell=j+1$,

$$
\begin{aligned}
\left\|\left(R_{h, 1} f\right)^{\prime}\right\|_{L_{p}(0, L)}^{p} & =\sum_{j=0}^{n-1} h_{j}^{1-p} \int_{0}^{1}\left|c_{j} \psi^{(0)^{\prime}}(\xi)+c_{j+1} \psi^{(1)^{\prime}}(\xi)\right|^{p} d \xi \\
& \leqslant \int_{0}^{1}\left(\left|\psi^{(0)^{\prime}}\right|^{p^{\prime}}+\left|\psi^{(1)^{\prime}}\right|^{p^{\prime}}\right)^{p / p^{\prime}} d \xi \sum_{j=0}^{n-1} h_{j}^{1-p}\left(\left|c_{j}\right|^{p}+\left|c_{j+1}\right|^{p}\right) \\
& =C \sum_{j=0}^{n}\left(h_{j-1}^{1-p}+h_{j}^{1-p}\right)\left|c_{j}\right|^{p} \\
& \leqslant C \sum_{j=0}^{n}\left(h_{j-1}+h_{j}\right)^{1-p}\left|c_{j}\right|^{p}
\end{aligned}
$$


where $1 / p+1 / p^{\prime}=1$ and we have used in the last inequality, in the case $p>1$ only, the estimate

$$
h_{j-1}^{1-p}+h_{j}^{1-p} \leqslant 2(1+\gamma)^{p-1}\left(h_{j-1}+h_{j}\right)^{1-p},
$$

which is a consequence of (6.2). Thus we have obtained the estimate

$$
\left\|\left(R_{h, 1} f\right)^{\prime}\right\|_{L_{p}(0, L)} \leqslant C\left|T_{h}^{-1 / p^{\prime}} c\right|_{p}
$$

where again $|\cdot|_{p}$ is the usual $\ell_{p}$ norm in $\mathbb{C}^{n+1}$. The system (2.13) can be given the form

$$
T_{h}^{-1 / p^{\prime}} A_{h} T_{h}^{1 / p^{\prime}}\left(T_{h}^{-1 / p^{\prime}} c\right)=T_{h}^{-1 / p^{\prime}} b,
$$

and Lemma 6.5 then implies the bound

$$
\left|T_{h}^{-1 / p^{\prime}} c\right|_{p} \leqslant C\left|T_{h}^{-1 / p^{\prime}} b\right|_{p}
$$

giving, with (2.15),

$$
\left\|\left(R_{h, 1} f\right)^{\prime}\right\|_{L_{p}(0, L)}^{p} \leqslant C \sum_{j=0}^{n}\left(h_{j-1}+h_{j}\right)^{1-p}\left|b_{j}\right|^{p}=C \sum_{j=0}^{n} \frac{\left|\left(f, \psi_{j}\right)_{h}\right|^{p}}{\left(h_{j-1}+h_{j}\right)^{2 p-1}} .
$$

Finally, we can prove in the same way as in the middle part of the proof of Lemma 4.2 that the right-hand side of (6.13) is bounded by $C\left\|D_{h} f\right\|_{h, p}^{p}$, proving (6.7).

\section{IMPOSING ZERO BOUNDARY CONDITIONS}

In this last section we discuss the case that the space $S_{h}$ is replaced by

$$
S_{h}^{0}:=\left\{v_{h} \in S_{h}: v_{h}(0)=v_{h}(L)=0\right\} .
$$

By $R_{h}^{0}: G_{h} \rightarrow S_{h}^{0}$ we denote the map corresponding to $R_{h}: G_{h} \rightarrow S_{h}$ defined by

$$
\left(R_{h}^{0} f, \psi\right)_{h}=(f, \psi)_{h} \quad \forall \psi \in S_{h}^{0} .
$$

As we shall see, $R_{h}^{0}$ shares many properties with $R_{h}$ but there are also some differences. The first one is that we obtain in comparison to Proposition 2.1 now the following characterisation that $(.,,)_{h}$ is an inner product on $S_{h}^{0}$.

Proposition 7.1. The positive semidefinite Hermitian form $(., .)_{h}$ is an inner product on $S_{h}^{0}$ if and only if either $J \geqslant r$, or $J=r-1$ and at least one of the extreme quadrature points $\xi_{1}$ or $\xi_{J}$ lies in $(0,1)$.

Proof: Assume $J=r-1$ and $\xi_{1} \in(0,1)$. Again $\|\psi\|_{h, 2}=0$ implies (2.1). Since $\psi(0)=0$ it follows that $\psi$ has $r$ roots in $\left[x_{0}, x_{1}\right]$ and hence $\psi=0$ in $\Delta_{0}$. Due to the continuity of $\psi$ it follows that $\psi\left(x_{1}\right)=0$. The reasoning can then be repeated for the interval $\left[x_{1}, x_{2}\right]$ and so forth, showing that $\psi=0$. If $\xi_{J} \in(0,1)$ one starts with $\left[x_{n-1}, x_{n}\right]$ 
first. The case $J \geqslant r$ is known already, so that we have shown that the conditions in the proposition are sufficient for $(.,)_{h}$ to be an inner product. The proof of the necessity, not spelt out here, follows from applying the elementary fact that a homogeneous linear system with fewer equations than unknowns has a non-trivial solution.

We assume in the sequel that $(.,)_{h}$ is an inner product on $S_{h}^{0}$. In the present case we can give more criteria than in Theorem 4.4 for the stability of $\left\{R_{h}^{0}\right\}_{H}$.

THEOREM 7.2. The sequence $\left\{R_{h}^{0}\right\}_{H}$ is $p$-stable for $p \in[1, \infty]$ if one of the following conditions hold:

$$
\sigma>0 \text { in (3.2) }
$$
any of the conditions (a)-(f) in Proposition 3.3 holds

$J \geqslant r$ and the partition $\pi_{h}^{\prime}$ is uniform

$$
\begin{aligned}
& J=r-1, \xi_{1}>0, \xi_{J}=1 \text { and } \inf \left\{\alpha_{k}: k=1, \cdots, n-1, h \in H\right\}>0 \\
& J=r-1, \xi_{1}=0, \xi_{J}<1 \text { and } \sup \left\{\alpha_{k}: k=1, \cdots, n-1, h \in H\right\}<1 .
\end{aligned}
$$

Proof: Corresponding to (2.4) we split

$$
S_{h}^{0}=S_{h, 1}^{0} \dot{+} S_{h, 2},
$$

where $S_{h, 2}$ is defined in (2.5) and $S_{h, 1}^{0}=S_{h}^{0} \cap S_{h, 1}$. The functions $\psi_{1}, \cdots, \psi_{n-1}$ form a basis in $S_{h, 1}^{0}$. In place of $A_{h}$ we obtain an $(n-1) \times(n-1)$ matrix $A_{h}^{0}$ with elements

$$
A_{k \ell}^{0}=A_{k \ell}, \quad k, \ell=1, \cdots, n-1,
$$

where $A_{k \ell}$ is given by (2.14). The $p$-stability of $\left\{R_{h}^{0}\right\}_{H}$ is then inferred from the inverse $\infty$-stability of $\left\{A_{h}^{0}\right\}_{H}$ as in Lemma 4.2. Define $A_{1,0}^{0}:=A_{n-1, n}^{0}:=0$ and

$$
\sigma_{h, k}^{0}:=A_{k k}^{0}-\left|A_{k, k-1}^{0}\right|-\left|A_{k, k+1}^{0}\right|, k=1, \cdots, n-1 .
$$

It is clear that with $\sigma_{i, k}$ given by (3.1)

$$
\sigma_{h, k}^{0} \geqslant \sigma_{h, k}, k=1, \cdots, n-1 .
$$

So the proof of Proposition 3.9 also gives the inverse $\infty$-stability of $\left\{A_{h}^{0}\right\}_{H}$, assuming (7.3) or (7.4) to hold. In the case of (7.5) we have $\alpha_{k}=1 / 2, k=1, \cdots, n-1$, and from (3.1) and (7.8) we obtain

$$
\sigma_{h, k}^{0} \geqslant \frac{1}{2} Q\left(\left|\psi^{(1)}-\psi^{(0)} \operatorname{sgn} Q\left(\psi^{(1)} \psi^{(0)}\right)\right|^{2}\right), k=1, \cdots, n-1 .
$$

The latter quantity is positive, as we have shown in the first part of the proof of Proposition 3.3. 
Now assume (7.7) to hold; the proof in the case of (7.6) is similar. With a similar argument to that used to prove Proposition $3.3(\mathrm{e})$ it can be shown that

$$
\sigma_{h, k}=\left(1-\alpha_{k}\right) w_{1}>0, \quad k=0, \cdots, n,
$$

from which follows with (7.7) and (7.8) that

$$
\sigma_{h, k}^{0} \geqslant\left(1-\sup \alpha_{k}\right) w_{1}>0, k=1, \cdots n-1 \text {. }
$$

THEOREM 7.3. Let $p \in[1, \infty]$ and let $\left\{R_{h}^{0}\right\}_{H}$ be $p$-stable. Then

$$
\begin{aligned}
& \left\|R_{h}^{0} g-g\right\|_{L_{s}(0, L)} \rightarrow 0(h \in \dot{H}), g \in C_{0}[0, L] \\
& \left\|R_{h}^{0} g-g\right\|_{L_{p}(0, L)} \leqslant C h_{\max }^{\ell}\left\|g^{(\ell)}\right\|_{L_{p}(0, L)}, g \in \dot{\mathfrak{W}}_{p}^{\ell}(0, L),
\end{aligned}
$$

for $\ell=1, \cdots, r$. Here

$$
C_{0}[0, L]:=\{g \in C[0, L]: g(0)=g(L)=0\}
$$

and

$$
\stackrel{\circ}{W}_{p}^{\ell}(0, L):=\left\{g \in W_{p}^{\ell}(0, L): g(0)=g(L)=0\right\} .
$$

Proof: The same reasoning as in the proof of Theorem 5.1 applies. The interpolating function $g_{h} \in S_{h}$ used there is now, due to the zero boundary conditions on $g$, an element of $S_{h}^{0}$.

The map $R_{h}^{0}$ is well-defined and $p$-stable in the case $J=r-1$ if the additional conditions given in Theorem 7.2 are satisfied; see (7.6) and (7.7). One might conjecture that $p$-stability holds also for symmetric quadrature rules when $J=r-1$, but as our final result we show that this is not the case.

Proposition 7.4. Let $J=r-1, \xi_{1}>0$ and the quadrature rule $Q$ be symmetric. Then for all $p \in[1, \infty],\left\{R_{h}^{0}\right\}_{H}$ is not $p$-stable.

PROOF: We construct a null-sequence $\left\{f_{h}\right\}_{H}$ of grid functions such that for all $p \in$ $[0, \infty],\left\{R_{h, 1}^{0} f_{h}\right\}_{H}$ does not converge to zero. Define

$$
f_{h}:=\sum_{k=1}^{n-1} c_{k} \psi_{k}, c_{k}:=(-\tau)^{n-k} \sin \left(\frac{\pi}{L} x_{k}\right)
$$

where

$$
\tau:=\operatorname{sgn}\left(Q\left(\psi^{(1)} \psi^{(0)}\right)\right)
$$

Since

$$
f_{h} \mid \Delta_{k}=c_{k} \psi_{k}+c_{k+1} \psi_{k+1}, \quad k=1, \cdots, n-2
$$


it follows that for $k=1, \cdots, n-2$ and $j=1, \cdots, J$

$$
\begin{aligned}
f_{h}\left(x_{k, j}\right)= & (-\tau)^{n-k-1}\left(-\tau \psi^{(0)}\left(\xi_{j}\right) \sin \left(\frac{\pi}{L} x_{k}\right)+\psi^{(1)}\left(\xi_{j}\right) \sin \left(\frac{\pi}{L} x_{k+1}\right)\right) \\
= & (-\tau)^{n-k-1}\left[\left(\psi^{(1)}\left(\xi_{j}\right)-\tau \psi^{(0)}\left(\xi_{j}\right)\right) \sin \left(\frac{\pi}{L} x_{k+1}\right)\right. \\
& \left.-\tau \psi^{(0)}\left(\xi_{j}\right)\left(\sin \left(\frac{\pi}{L} x_{k}\right)-\sin \left(\frac{\pi}{L} x_{k+1}\right)\right)\right] \\
= & (-\tau)^{n-k} \psi^{(0)}\left(\xi_{j}\right)\left(\sin \left(\frac{\pi}{L} x_{k}\right)-\sin \left(\frac{\pi}{L} x_{k+1}\right)\right),
\end{aligned}
$$

where in the last step Lemma 7.5 below has been taken into account. Hence, for $k=$ $1, \cdots, n-2$

$$
\left|f_{h}\left(x_{k, j}\right)\right| \leqslant C h_{k}, \quad j=1, \cdots, J .
$$

It is easily seen that (7.12) also holds for $k=0$ and $k=n-1$, and consequently

$$
\left\|f_{h}\right\|_{h, p} \rightarrow 0 \quad(h \in H) .
$$

On the other hand the vector $c_{h}:=\left(0, c_{1}, \cdots, c_{n-1}, 0\right) \in \mathbb{C}^{n+1}$ satisfies

$$
\begin{aligned}
\left|c_{h}\right|_{h, p} & =\left(\sum_{k=1}^{n-1} \frac{h_{k-1}+h_{k}}{2}\left|\sin \left(\frac{\pi}{L} x_{k}\right)\right|^{p}\right)^{1 / p} \\
& \rightarrow\left(\int_{0}^{L}\left|\sin \left(\frac{\pi}{L} x\right)\right|^{p} d x\right)^{1 / p}>0 \quad(h \in H) .
\end{aligned}
$$

Since $f_{h} \in S_{h, 1}^{0}$ we have $R_{h, 1}^{0} f_{h}=f_{h}$. Hence, with the aid of the inequality

$$
|c|_{h, p} \leqslant C\left\|\sum_{k=0}^{n} c_{k} \psi_{k}\right\|_{L_{p}(0, L)}, \quad c \in \mathbb{C}^{n+1},
$$

(which is obtained by scaling the $L_{p}$-integrals over the subintervals $\triangle_{k}$ and using the equivalence of all norms in two-dimensional spaces) we conclude that

$$
\left\|R_{h, 1}^{0} f_{h}\right\|_{L_{p}(0, L)}=\left\|f_{h}\right\|_{L_{p}(0, L)} \nrightarrow 0 \quad(h \in H),
$$

which shows the instability of $\left\{R_{h, 1}^{0}\right\}_{H}$. Then also $\left\{R_{h}^{0}\right\}_{H}$ is not stable since we have proved in Lemma 4.1 that $\left\{R_{h, 2}^{0}\right\}_{H}=\left\{R_{h, 2}\right\}_{H}$ is always stable.

It remains only to prove the following lemma.

Lemma 7.5. Let the assumptions of Proposition 7.4 hold. Then

$$
\psi^{(1)}\left(\xi_{j}\right)=\psi^{(0)}\left(\xi_{j}\right) \operatorname{sgn}\left(Q\left(\psi^{(1)} \psi^{(0)}\right)\right) \quad j=1, \cdots, J
$$

PROOF: Since there are $r-1$ interior nodes the sesquilinear form

$$
Q(x(1-x) \psi \bar{\phi}), \quad \phi, \psi \in \mathbb{P}_{r-2},
$$


defines an inner product on $\mathbb{P}_{r-2}$. Let $0 \neq q_{r-2}$ be a real polynomial which is orthogonal to $\mathbb{P}_{r-3}$ with respect to (7.14). From the defining equations (2.7) of $\psi^{(i)}$ we obtain

$$
Q\left(x(1-x) \psi^{(i)} \bar{\phi}\right)=0, \quad \phi \in \mathbb{P}_{r-3}, \quad i=0,1 .
$$

Thus the vector obtained by restricting $\psi^{(i)}$ to the points $\xi_{j}, j=1, \cdots, r-1$, satisfies the same orthogonality conditions as $q_{r-2}$. Since the orthogonal complement of $\mathbb{P}_{r-3}$ with respect to (7.14) is one-dimensional, the relation

$$
\psi^{(i)}\left(\xi_{j}\right)=\beta_{i} q_{r-2}\left(\xi_{j}\right), \quad j=1, \cdots, r-1, \quad i=0,1,
$$

for some real constants $\beta_{0}$ and $\beta_{1}$ follows. The symmetry of $Q$ implies $Q\left(\psi^{(1) 2}\right)=$ $Q\left(\psi^{(0) 2}\right)$, and we conclude $\left|\beta_{0}\right|=\left|\beta_{1}\right|$. The assertion follows now by straightforward calculation using (7.15).

In passing we remark also that Theorem 6.1 holds if $R_{h}$ is replaced by $R_{h}^{0}$.

\section{A. APPENDIX}

For the convenience of the reader we provide the approximation properties of $S_{h}$ needed in Section 5.

Lemma A.1. Let $p \in[1, \infty]$ and $g \in W_{p}^{1}(0, L)$. Then the piecewise linear interpolant $g_{h}$ of $g$ interpolating at the breakpoints $x_{0}, \cdots, x_{n}$ satisfies

$$
\begin{aligned}
\left\|g-g_{h}\right\|_{L_{p}(0, L)} & \leqslant C h_{\max }\left\|g^{\prime}\right\|_{L_{p}(0, L)} \\
\left\|g-g_{h}\right\|_{h, p} & \leqslant C h_{\max }\left\|g^{\prime}\right\|_{L_{p}(0, L)} .
\end{aligned}
$$

Proof: Let $G$ denote the Green's function for $d^{2} / d x^{2}$ with Dirichlet boundary conditions at $x=0$ and $x=1$. Then, if $f \in C^{2}[\dot{0}, 1]$ and $f_{I}$ is the linear function interpolating $f$ at 0 and 1 , we have the representation

$$
\begin{aligned}
f(x)-f_{I}(x) & =\int_{0}^{1} G(x, y) f^{\prime \prime}(y) d y \\
& =-\int_{0}^{1} G_{y}(x, y) f^{\prime}(y) d y, \quad x \in[0,1] .
\end{aligned}
$$

(It can be verified directly that this identity holds also for $f \in W_{\infty}^{1}(0, L)$.) It follows with Hölder's inequality that

$$
\left\|f-f_{I}\right\|_{L_{\infty}(0,1)} \leqslant \sup _{x \in(0,1)}\left(\int_{0}^{1}\left|G_{y}(x, y)\right|^{p^{\prime}} d y\right)^{1 / p^{\prime}}\left\|f^{\prime}\right\|_{L_{p}(0,1)} .
$$

In the usual way we obtain by scaling and taking $f(x):=g\left(x_{k}+h_{k} x\right)$

$$
\left\|g-g_{h}\right\|_{L_{\infty}\left(\Delta_{k}\right)} \leqslant C h_{k}^{1-1 / p}\left\|g^{\prime}\right\|_{L_{p}\left(\Delta_{k}\right)}, \quad k=0, \cdots, n-1,
$$


and hence

$$
\begin{aligned}
\left\|g-g_{h}\right\|_{L_{p}\left(\Delta_{k}\right)}^{p} & \leqslant C h_{k}^{p}\left\|g^{\prime}\right\|_{L_{p}\left(\Delta_{k}\right)}^{p}, \\
h_{k} \sum_{j=1}^{J} w_{j}\left|\left(g-g_{h}\right)\left(x_{k, j}\right)\right|^{p} & \leqslant C h_{k}^{p}\left\|g^{\prime}\right\|_{L_{p}\left(\triangle_{k}\right)}^{p} .
\end{aligned}
$$

Summation with respect to $k$ proves the assertions.

LemmA A. 2. Let $p \in[1, \infty]$ and $2 \leqslant \ell \leqslant r$. Then, for each $g \in W_{p}^{\ell}(0, L)$ there exists a function $g_{h} \in S_{h}$ interpolating $g$ at the breakpoints $x_{0}, \cdots, x_{n}$ which satisfies

$$
\begin{aligned}
\left\|g-g_{h}\right\|_{L_{p}(0, L)} & \leqslant C h_{\max }^{\ell}\left\|g^{(\ell)}\right\|_{L_{p}(0, L)}, \\
\left\|g-g_{h}\right\|_{h, p} & \leqslant C h_{\max }^{\ell}\left\|g^{(\ell)}\right\|_{L_{p}(0, L)}
\end{aligned}
$$

Proof: Choose a set of boundary conditions $B_{k}, k=1, \cdots, \ell$, at $x=0$ and $x=1$ containing derivatives of order $\ell-1$ at most, such that

$$
B_{1} f=f(0), \quad B_{2} f=f(1)
$$

and such that the condition

$$
f \in \mathbb{P}_{\ell-1}, B_{k} f=0, k=1, \cdots, \ell \Rightarrow f=0
$$

holds. Denote by $G$ the Green's function of $(d / d x)^{\ell}$ subject to the corresponding homogeneous boundary conditions. For $f \in W_{p}^{\ell}(0,1)$ choose $f_{I} \in \mathbb{P}_{\ell-1}$ such that

$$
B_{k}\left(f-f_{I}\right)=0, k=1, \cdots, \ell \text {. }
$$

We then have the representation

$$
f(x)-f_{I}(x)=\int_{0}^{1} G(x, y) f^{(\ell)}(y) d y, \quad x \in(0,1)
$$

and obtain by an application of Hölder's inequality

$$
\left\|f-f_{I}\right\|_{L_{\infty}(0,1)} \leqslant \sup _{x \in(0,1)}\left(\int_{0}^{1}|G(x, y)|^{p^{\prime}} d y\right)^{1 / p^{\prime}}\left\|f^{(\ell)}\right\|_{L_{p}(0,1)} .
$$

The function $g_{h}$ is constructed by defining, on each subinterval $\Delta_{k}$, a polynomial in $\mathbb{P}_{\ell-1}$ corresponding to the first part of the proof; this polynomial interpolates $g$ at the endpoints of $\triangle_{k}$. Patching the pieces together, a function $g_{h} \in S_{h}$ is obtained. By a scaling argument one derives from (A1) the bound

$$
\left\|g-g_{h}\right\|_{L_{\infty}\left(\Delta_{k}\right)} \leqslant C h_{k}^{\ell-1 / p}\left\|g^{(\ell)}\right\|_{L_{p}\left(\Delta_{k}\right)}, k=0, \cdots, n-1
$$

The assertion is then proved as in the last part of the proof of Lemma Al. 


\section{REFERENCES}

[1] C. de Boor, 'A bound on the $L^{\infty}$-norm of $L^{2}$-approximation by splines in term of a global mesh ratio', Math. Comp. 30 (1976), 765-771.

[2] M. Crouzeix and V. Thomée, 'The stability in $L_{p}$ and $W_{p}^{1}$ of the $L_{2}$-projection onto finite element function spaces', Math. Comp. 48 (1987), 521-532.

[3] P.J. Davis and P. Rabinowitz, Methods of numerical integration (Academic Press, New York, 1975).

[4] J. Descloux, 'On finite element matrices', SIAM J. Numer. Anal. 9 (1972), 260-265.

[5] J. Douglas, T. Dupont and L. Wahlbin, 'Optimal $L_{\infty}$ error estimates for Galerkin approximations to solutions of two-point boundary value problems', Math. Comp. 29 (1975), 475-483.

[6] J. Douglas, T. Dupont and L. Wahlbin, 'The stability in $L^{q}$ of the $L^{2}$-projection into finite element subspaces', Numer. Math. 23 (1975), 193-197.

[7] R. Dautray and J.-L. Lions, Mathematical analysis and numerical methods for science and technology 4 (Springer-Verlag, Berlin, Heidelberg, New York, 1990).

[8] M. Ganesh and I. H. Sloan, 'Optimal order spline methods for nonlinear differential and integro-differential equations', Appl. Numer. Math. (to appear).

[9] R.D. Grigorieff and I.H. Sloan, 'Spline Petrov-Galerkin methods with quadrature', $\mathrm{Nu}$ mer. Funct. Anal. Optim. 17 (1996), 755-784.

[10] R.D. Grigorieff and I.H. Sloan, 'High-order spline Petrov-Galerkin methods with quadrature', in Proceedings of the International Congress on Industrial and Applied Mathematics Hamburg, 1995,, (G. Alefeld et al., Editors) (Z. Angew Math. Mech. 76, 1996), pp. 15-18.

[11] L. Wahlbin, Superconvergence in Galerkin Finite Element Methods, Lecture Notes in Mathematics 1605 (Springer, Berlin, 1995).

Technische Universität Berlin

Strasse des 17. Juni 135

D-10623 Berlin

Germany

e-mail: grigo@math.tu-berlin.de
School of Mathematics

University of New South Wales

Sydney 2052

Australia

e-mail: I.Sloan@unsw.edu.au 